\title{
A Non-Conventional Coloring of the Edges of a Graph
}

\author{
Sándor Szabó \\ Department of Applied Mathematics, Institute of Mathematics and Informatics, \\ University of Pécs, Pécs, Hungary \\ Email: sszabo7@hotmail.com
}

Received August 15, 2012; revised September 3, 2012; accepted September 15, 2012

\begin{abstract}
Coloring the nodes of a graph is a commonly used technique to speed up clique search algorithms. Coloring the edges of the graph as a preconditioning method can also be used to speed up computations. In this paper we will show that an unconventional coloring scheme of the edges leads to an NP-complete problem when one intends to determine the optimal number of colors.
\end{abstract}

Keywords: Maximum Clique; Coloring the Vertices of a Graph; Coloring the Edges of Graph; NP-Complete Problems

\section{Introduction}

Let $G=(V, E)$ be a finite simple graph. This means that $G$ has finitely many nodes, that is, $|V|$ is finite. Further $G$ does not have any double edge or loop. In this special case an edge of $G$ can be identified with a two element subset of $V$. As a consequence the set of edges $E$ of $G$ is a family of two element subsets of $V$. A subgraph $\Delta$ of $G$ is called a clique if two distinct nodes of $\Delta$ are always adjacent in $G$. A clique with $k$ nodes is simply called a $k$-clique. The number of nodes of a clique is sometimes referred as the size of the clique. A 1-clique in $G$ is a vertex of $G$. A 2-clique in $G$ is an edge in $G$. A 3-clique in $G$ is sometimes referred as a triangle in $G$. A $k$-clique in $G$ is called a maximal clique if it is not a subgraph of any $(k+1)$-clique in $G$. A $k$-clique in $G$ is defined to be a maximum clique if $G$ does not contain any $(k+1)$-clique. The graph $G$ may have several maximum cliques. However, each maximum clique in $G$ has the same number of nodes. This well defined number is called the clique size of $G$ and it is denoted by $\omega(G)$.

Determining the size of a maximum clique in a given graph is an important problem in applied and pure discrete mathematics. A number of selected applications are presented in [1] and [2]. It was recognized in [3] that the efficiency of the clique search algorithms can be crucially improved by coloring and rearranging the nodes of the tested graph. In [4] instead of coloring the nodes a technique based on dynamic programming was used successfully. In [5] another coloring idea was presented. This time the edges of the graph were colored. Numerical evidence shows that the edge coloring provides sharper upper bounds for the clique size than the node coloring.
However, the improved upper bound comes for a higher computational cost.

We color the nodes of a finite simple graph $G=(V, E)$ using $k$ colors. We assume that the coloring satisfies the following conditions: 1) Each node of $G$ receives exactly one color; 2) Adjacent nodes in $G$ never receive the same color. We will call this an $L$ type coloring of the nodes of $G$ with k colors. The letter $L$ refers to the expression legal coloring. We may use the numbers $1, \cdots, k$ as colors. The coloring can be defined by a map $f: v \rightarrow\{1, \cdots, k\}$ which is onto. Provided that

$f\left(v_{1}\right)=f\left(v_{2}\right), v_{1} \neq v_{2}$ imply that $v_{1}$ and $v_{2}$ are not adjacent for each $v_{1}, v_{2} \in V$.

We color the edges of a finite simple graph $G=(V, E)$ using $k$ colors. We suppose that for the coloring the next two conditions hold: 1) Each edge of $G$ receives exactly one color; 2) If $x, y, u, v$ are nodes of a 4-clique in $G$, then the edges $\{x, y\}$ and $\{u, v\}$ cannot have the same color. We will call this a $B$ type coloring of the edges of $G$ with $k$ colors. In a conversation Bogdán Zaválnij proposed this edge coloring scheme. The letter $B$ comes from the name Bogdán. The coloring can be described by a map $g: E \rightarrow\{1, \cdots, k\}$ that is onto. We require that $g(\{x, y\})=g(\{u, v\})$, and $\{x, y\} \cap\{u, v\}=\varnothing$ imply that $x, y, u, v$ are not nodes of a 4-clique in $G$ for each $\{x, y\},\{u, v\} \in E$.

Problem 1. Given a finite simple graph $G$ and given a positive integer $k$. Decide if the edges of $G$ have $B$ type coloring using $k$ colors.

The smaller is the number of colors $k$ for which the edges of $G$ have a $B$ type coloring the more useable is the coloring in connection with clique search. The main result of this paper is that Problem 1 is NP-complete for 
$k=3$. The intuitive meaning of this result is that determining the threshold value of the colors $k$ in Problem 1 is computationally demanding. Consequently, in practical computations we have to resort on approximate greedy algorithms and we have to develop various heuristics.

\section{The Auxiliary Graph $H$}

In this section we construct an auxiliary graph. This will play the role of building blocks in further constructions. Let us consider the graph $H=(V, E)$ given by its adjacency matrix in Table 1. The graph $H$ has 14 nodes $v_{1}, \cdots, v_{14}$ and 24 edges. The rows and columns of the adjacency matrix of $\mathrm{H}$ are labeled by the nodes. The bullet in the cell at the intersection of row $v_{i}$ and column $v_{j}$ records the fact that the unordered pair $\left\{v_{i}, v_{j}\right\}$ is an edge of $H$. The reader will notice that in Table 1 the node $v_{i}$ is replaced by $i$, that is, the letter $v$ is suppressed and only the index $i$ is used. In Table 2 each node and its neighbors are listed. This is another way to describe the graph $H$. The set of neighbors of the node $v$ in $G$ is denoted by $N(v)$ and by definition $N(v)=\{x:\{v, x\} \in E, x \in V\}$. Finally, the geometric representation of the graph $H$ is given in Figure 1. Right below each node we recorded the color of the node. But at this moment the reader may ignore the colors. In order to avoid a cluttered figure we used two copies of the nodes $v_{1}$ and $v_{14}$. One can imagine that the figure is drawn on a strip of paper. Then we fold the strip to form a cylinder identifying the shorter sides of the strip. Thus the graph $H$ is drawn on the surface of a cylinder and we arrange things such that the two copies of $v_{1}$ coincide

Table 1. The adjacency matrix of graph $H$.

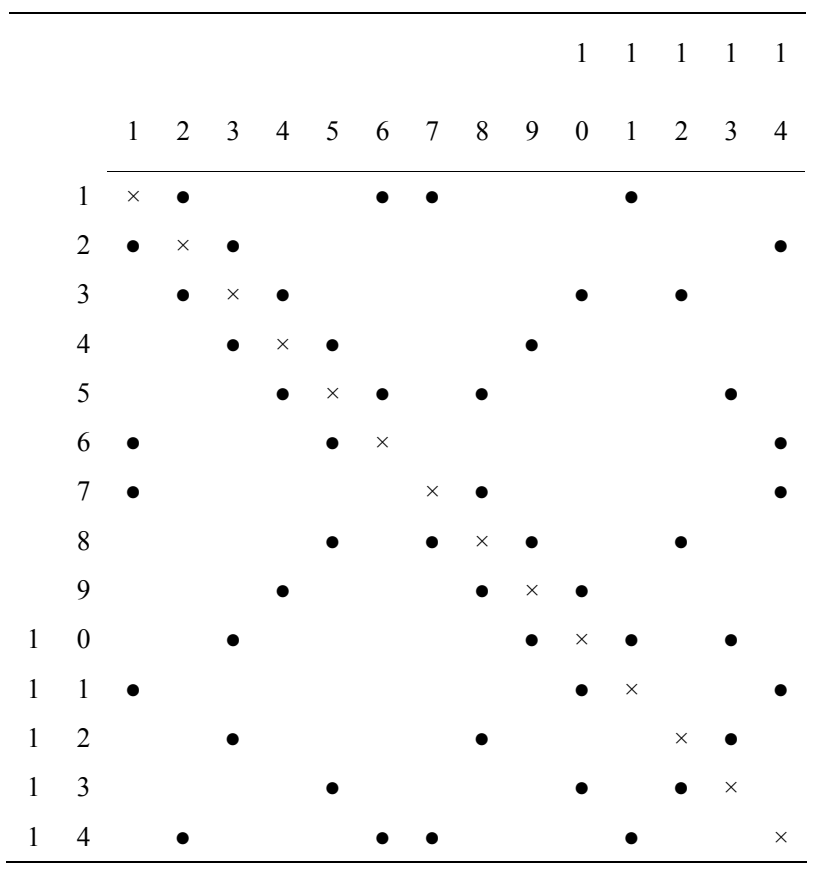

Table 2. The lists of the neighbors of the nodes in $H$.

\begin{tabular}{l|llll}
\hline 1 & 2 & 6 & 7 & 11 \\
2 & 1 & 3 & 14 & \\
3 & 2 & 4 & 10 & 12 \\
4 & 3 & 5 & 9 & \\
5 & 4 & 6 & 8 & 13 \\
6 & 1 & 5 & 14 & \\
7 & 1 & 8 & 14 & \\
8 & 5 & 7 & 9 & 12 \\
9 & 4 & 8 & 10 & \\
10 & 3 & 9 & 11 & 13 \\
11 & 1 & 10 & 14 & \\
12 & 3 & 8 & 13 & \\
13 & 5 & 10 & 12 & \\
14 & 2 & 6 & 7 & 11 \\
\hline
\end{tabular}

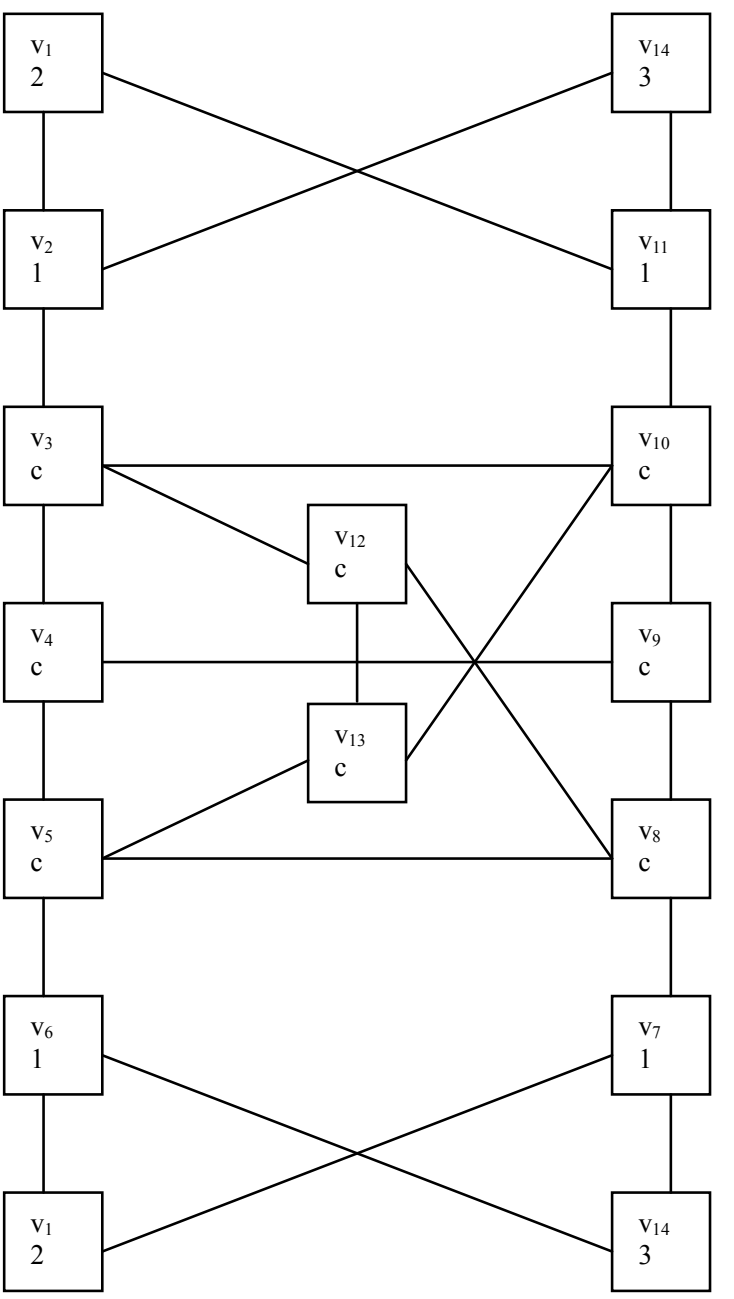

Figure 1. Graph $\boldsymbol{H}$ with colored nodes. 
and also the two copies of $v_{14}$ coincide. The properties of the graph $H$ we will use later are spelled out formally as a proposition.

Proposition 1. (1) The graph $H$ does not contain any 3-clique. (2) In an $L$ type coloring of the nodes of $H$ with 3 colors the nodes $v_{1}$ and $v_{14}$ must receive the same color. (3) The nodes of $H$ do have an $L$ type coloring with 3 colors.

Proof. The statements of the proposition can be verified by simple inspections. Here is how the inspection goes in connection with statement (1). Pick the bullet in the cell at the intersection of row $v_{1}$ and column $v_{2}$. This bullet represents the edge $\left\{v_{1}, v_{2}\right\}$. Scanning the rows $v_{1}$ and $v_{2}$ of the adjacency matrix we can see that $N\left(v_{1}\right) \cap N\left(v_{2}\right)=\varnothing$. This means that the edge $\left\{v_{1}, v_{2}\right\}$ cannot be a side of any triangle in $H$. Then repeat the argument for all the 24 edges of $H$.

In order to prove the statement (2) let us assume on the contrary that there is an $L$ type coloring $f: V \rightarrow\{1,2,3\}$ of the nodes of $H$ such that $f\left(v_{1}\right) \neq f\left(v_{14}\right)$. We may assume that $f\left(v_{1}\right)=2$ and $f\left(v_{14}\right)=3$ since this is only a matter of rearranging the colors 1, 2, 3 among each other. Note that $f\left(v_{1}\right)$ and $f\left(v_{11}\right)$ must be equal to 1. From this it follows that $f\left(v_{3}\right) \in\{2,3\}$ and

$f\left(v_{10}\right) \in\{2,3\}$. A similar argument gives that

$f\left(v_{6}\right), f\left(v_{7}\right)$ must be equal to 1 and $f\left(v_{5}\right) \in\{2,3\}$,

$f\left(v_{8}\right) \in\{2,3\}$. This portion of the reasoning can be followed in Figure 1. We distinguish four cases listed in Table 3. Let us consider case 1. As $f\left(v_{3}\right)=2$ and $f\left(v_{8}\right)=2$, it follows that $f\left(v_{12}\right)=1$ must hold. Similarly, $f\left(v_{13}\right)=1$ must hold. But $v_{12}$ and $v_{13}$ are adjacent nodes in $\mathrm{H}$ and so we get the contradiction that $f\left(v_{12}\right) \neq f\left(v_{13}\right)$. The situation is illustrated in Figure 2. Let us consider case 2 . From $f\left(v_{3}\right)=2$ and

$f\left(v_{5}\right)=3$, it follows that $f\left(v_{4}\right)=1$ must hold. Similarly, $f\left(v_{9}\right)=1$ must hold. But $v_{4}$ and $v_{9}$ are adjacent nodes in $H$ and so we get the contradiction that $f\left(v_{4}\right) \neq f\left(v_{9}\right)$. Each of the remaining cases can be handled in an analogous way. The reasoning can be followed in Figure 3.

The statement (3) can be proved by exhibiting a required coloring. This is done in Figure 4.

\section{The Auxiliary Graph $K$}

Using the graph $H$ we construct a new graph $K$. Let

Table 3. The cases.

\begin{tabular}{ccccc}
\hline case & $f\left(v_{3}\right)$ & $f\left(v_{10}\right)$ & $f\left(v_{5}\right)$ & $f\left(v_{8}\right)$ \\
\hline 1 & 2 & 3 & 2 & 3 \\
2 & 2 & 3 & 3 & 2 \\
3 & 3 & 2 & 2 & 3 \\
4 & 3 & 2 & 3 & 2 \\
\hline
\end{tabular}

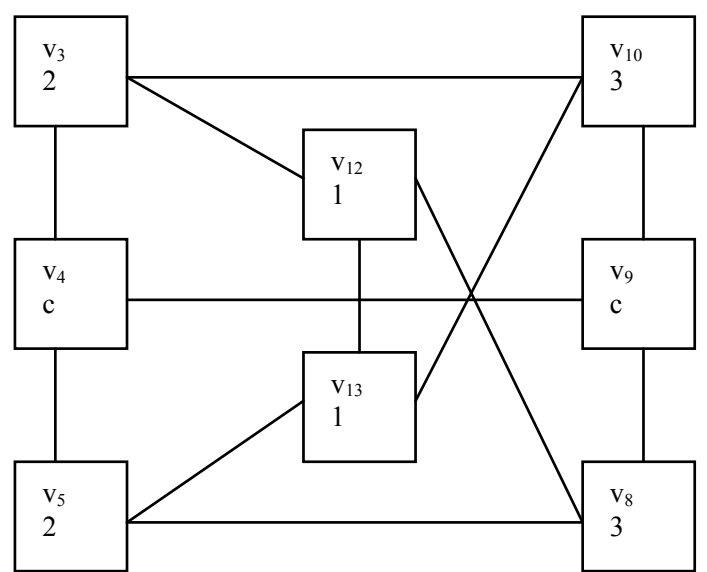

Figure 2. Case 1.

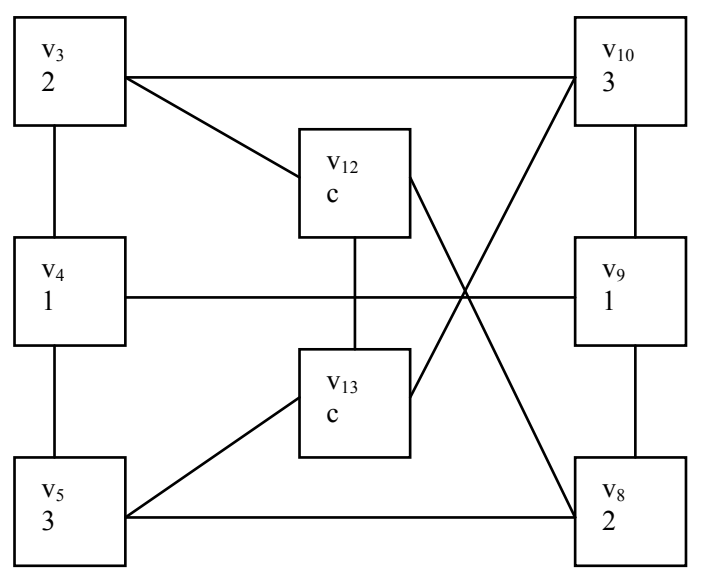

Figure 3. Case 2.

$x_{1}, \cdots, x_{14}, y_{1}, \cdots, y_{14}$ be pair-wise distinct points. These will be the nodes of $K$. We connect the nodes $x_{i}$ and $y_{i}$ for each $i, 1 \leq i \leq 14$. The edge $\left\{x_{i}, y_{i}\right\}$ of $K$ and the node $v_{i}$ of $H$ correspond to each other mutually. The edges $\left\{x_{i}, y_{i}\right\} \quad i, 1 \leq i \leq 14$ form a matching in $K$. In other words these edges do not have any common end points and their end points give all the nodes of $K$. We will call these edges of $K$ primary edges. We will call all the other edges of $K$ secondary edges. If $v_{i}$ and $v_{j}$ are adjacent nodes in $H$, then we add the following edges to $K$.

$$
\left\{x_{i}, x_{j}\right\} \quad\left\{x_{i}, y_{j}\right\} \quad\left\{y_{i}, x_{j}\right\} \quad\left\{y_{i}, y_{j}\right\}
$$

Figure 5 illustrates the construction. If $v_{i}$ and $v_{j}$ are not adjacent nodes in $H$, then we do not add any new edge to $K$. The new graph $K$ has $(2) \times(14)=28$ nodes and $(14)+[(24) \times(4)]=110$ edges.

To the node $v_{i}$ of $H$ we assigned the edge $\left\{x_{i}, y_{j}\right\}$ of $K$. To the edge $\left\{v_{i}, v_{j}\right\}$ of $H$ we assigned the 4-clique in $K$ whose nodes are $x_{i}, y_{i}, x_{j}, y_{j}$. Collapsing the nodes $x_{i}$ and $y_{j}$ to one point the edges (1) collapse to an edge and from $K$ we get back an isomor- 


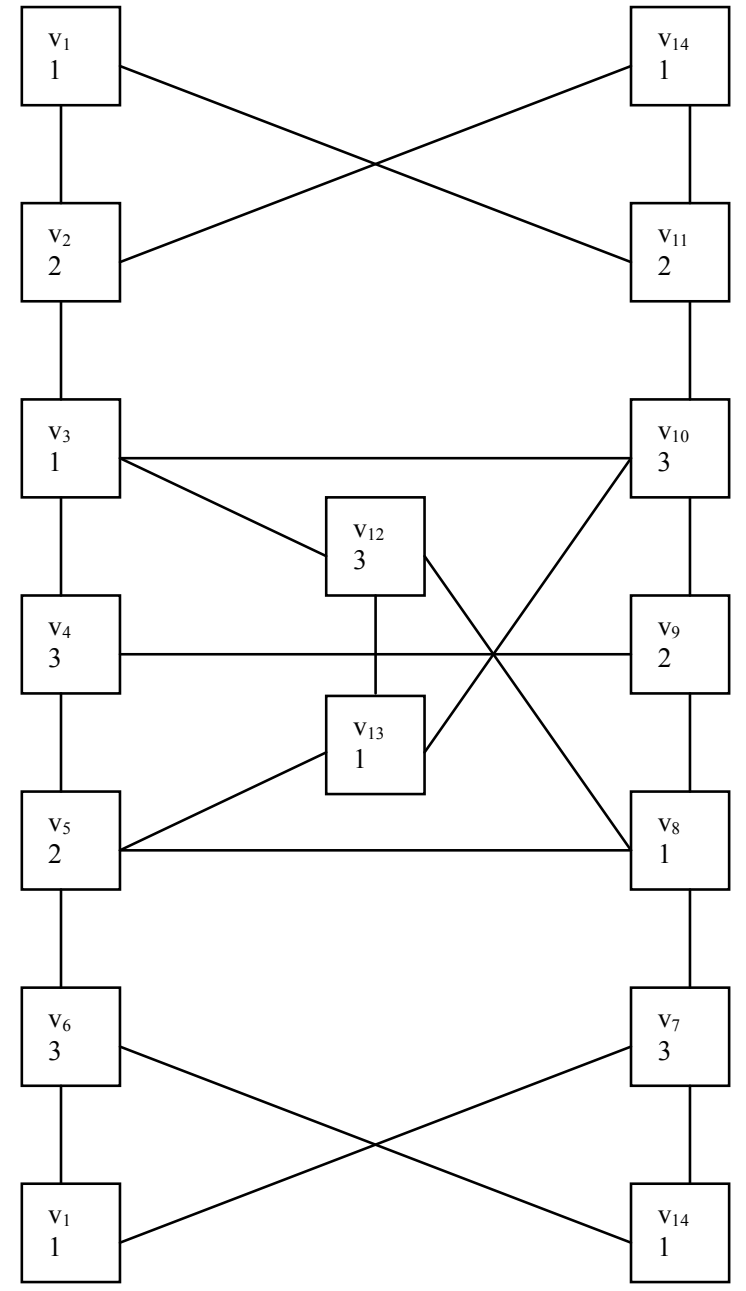

Figure 4. Graph $H$ with colored nodes.

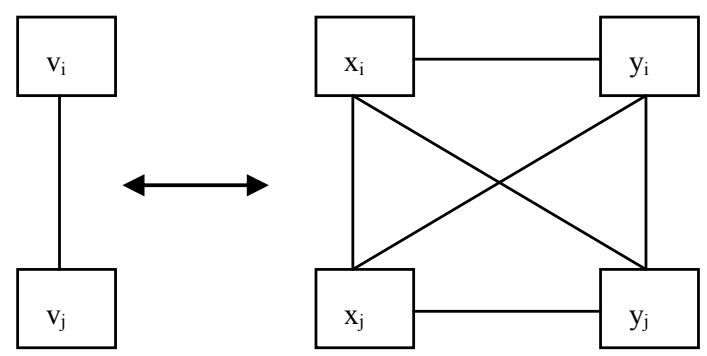

Figure 5. The correspondence between $H$ and $K$.

phic copy of $H$. We summarize the properties of $K$ we will need later in two propositions.

Proposition 2. (1) $\omega(K)=4$. (2) In each 4-clique in $K$ there are exactly two primary edges.

Proof. Clearly, $K$ contains a 4-clique. In order to prove statement (1) it is enough to verify that $K$ does not contain any 5-clique. We assume on the contrary that $K$ contains a 5-clique. As the primary edges form a matching in $K$, a 5-clique in $K$ can have only $0,1,2$ primary edges. The cases are depicted in Figures 6-8,

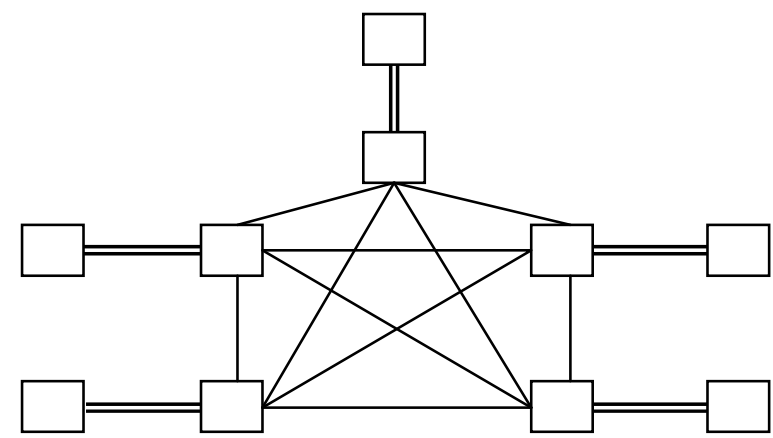

Figure 6. A 5-clique without primary edge.

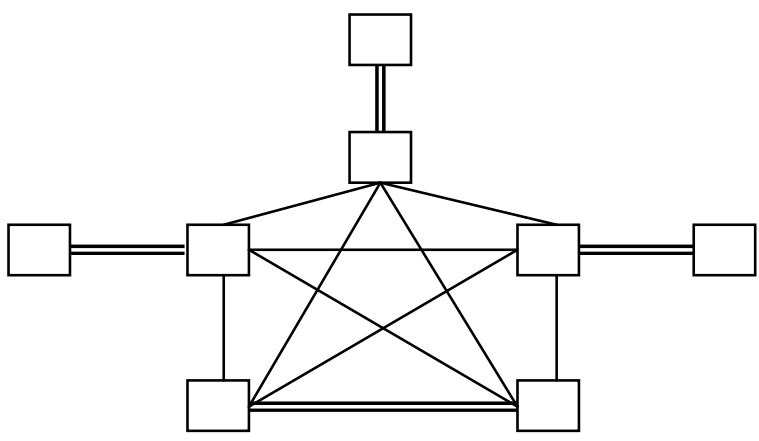

Figure 7. A 5-clique with one primary edge.

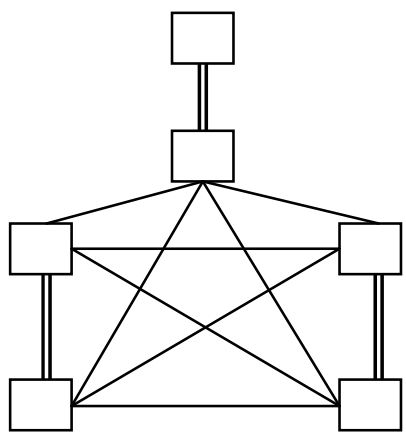

Figure 8. A 5-clique with two primary edges.

respectively. The primary edges are marked by double lines. Let us suppose first that the 5-clique does not have any primary edge. Then there are 5 primary edges joining to the 5-clique. From this it follows that $H$ must contain a 5-clique. When the 5-clique contains exactly one primary edge, then the graph $H$ must contain a 4-clique. Finally, when the 5-clique contains exactly two primary edges, then $H$ must contain a 3-clique. But by Proposition 1, $H$ does not contain any 3-clique. This contradiction proves statement (1). Statement (2) can be proved using the same technique.

Proposition 3. (1) The edges of $K$ have a $B$ type coloring with 3 colors. (2) In each such coloring of the edges of $K$ the edges $\left\{x_{1}, y_{1}\right\}$ and $\left\{x_{14}, y_{14}\right\}$ must receive the same color.

Proof. Let $f: V \rightarrow\{1,2,3\}$ be an $L$ type coloring 
of the nodes of $H=(V, E)$. By Proposition 1, such coloring exists. Using $f$ we can construct a $B$ type coloring $g: F \rightarrow\{1,2,3\}$ of the edges of $K=(W, F)$. We can achieve this by setting $g\left(\left\{x_{i}, y_{i}\right\}\right)$ to be equal to $f\left(v_{i}\right)$. If $v_{i}$ and $v_{j}$ are adjacent nodes in $H$, then the nodes $x_{i}, y_{i}, x_{j}, y_{j}$ are nodes of a 4-clique in $K$. Now $f\left(v_{i}\right) \neq f\left(v_{j}\right)$ and so $g\left(\left\{x_{i}, y_{i}\right\}\right) \neq g\left(\left\{x_{j}, y_{j}\right\}\right)$. The remaining four edges of the 4-clique we color in the following way. Set $g\left(\left\{x_{i}, y_{i}\right\}\right), g\left(\left\{x_{i}, y_{j}\right\}\right)$ to be equal to $f\left(v_{i}\right)$ and set $g\left(\left\{y_{i}, x_{j}\right\}\right), g\left(\left\{y_{i}, y_{j}\right\}\right)$ to be equal to $f\left(v_{j}\right)$. A routine inspection shows that the edges of the 4-clique whose nodes are $x_{i}, y_{i}, x_{j}, y_{j}$ have a $B$ type coloring with 3 colors. (The reader will notice that in fact we used only 2 colors to color the nodes of the 4-clique.) This coloring procedure can be repeated for each 4-clique in $K$ which has exactly two primary edges. By Proposition 2, each 4-clique in $K$ has exactly two primary edges and so each edge of $K$ is colored. Therefore the edges of $K$ have a $B$ type coloring with 3 colors. This proves statement (1).

In order to prove statement (2) let $g: F \rightarrow\{1,2,3\}$ be a $B$ type coloring of the edges of $K$. Using $g$ we can construct an $L$ type coloring $f: V \rightarrow\{1,2,3\}$

$f: V \rightarrow\{1,2,3\}$ of the nodes of $H$. Simply we set

$f\left(v_{i}\right) \quad f\left(v_{i}\right)$ to be equal to $g\left(\left\{x_{i}, y_{j}\right\}\right)$.

\section{The Auxiliary Graph $L$}

Let $K^{\prime}=\left(W^{\prime}, F^{\prime}\right)$ be an isomorphic copy of

$K=(W, F)$ such that the sets $W^{\prime}$ and $W$ are disjoint. Using $K$ and $K^{\prime}$ we construct a new graph $L$ by adding the following edges to $L$.

$$
\left\{x_{14},\left(x_{14}\right)^{\prime}\right\},\left\{x_{14},\left(y_{14}\right)^{\prime}\right\},\left\{y_{14},\left(x_{14}\right)^{\prime}\right\},\left\{y_{14},\left(y_{14}\right)^{\prime}\right\}
$$

Figure 9 illustrates the construction. These edges connect the graphs $K$ and $K^{\prime}$. The resulting graph is denoted by $L$. The graph $L$ has $(2) \times(28)=56$ nodes and $(4)+[(2) \times(110)]=224$ edges. The properties of the graph $L$ we will need later are spelled out in the next

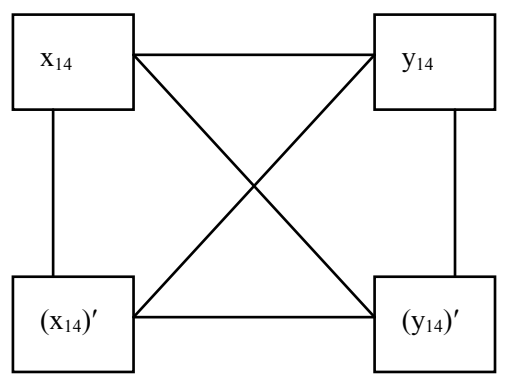

Figure 9. Connecting the graphs $K$ and $K^{\prime}$. proposition.

Proposition 4. (1) The edges of $L$ have $B$ type coloring with 3 colors. (2) In such a coloring of the edges of $L$ the edges $\left\{x_{1}, y_{1}\right\}$ and $\left\{x_{14}, y_{14}\right\}$ cannot receive the same color.

Proof. By Proposition 3, the edges of $K$ have a $B$ type coloring with 3 colors. In such a coloring of the edges of $K$ the edges $\left\{x_{1}, y_{1}\right\}$ and $\left\{x_{14}, y_{14}\right\}$ must receive the same color. Because the colors of the edges can be exchanged among each other freely we may in fact prescribe the colors of these edges. Similarly, the edges of $K^{\prime}$ have a $\mathrm{B}$ type coloring with 3 colors. In the coloring of the edges of $K^{\prime}$ the colors of the edges $\left\{\left(x_{1}\right)^{\prime},\left(y_{1}\right)^{\prime}\right\}$ and $\left\{\left(x_{14}\right)^{\prime},\left(y_{14}\right)^{\prime}\right\}$ must be equal. Again the color of these edges can be prescribed. Because of the presence of the edges (2) the edges $\left\{x_{14}, y_{14}\right\}$ and $\left\{\left(x_{14}\right)^{\prime},\left(y_{14}\right)^{\prime}\right\}$ must receive distinct colors.

\section{The Main Result}

We are ready to prove the main result of this paper.

Theorem 1. Problem 1 is NP-complete for $k=3$.

Proof. Let $G=(V, E)$ be a finite simple graph. Using $G$ we construct a new graph $G^{\prime}=\left(V^{\prime}, E^{\prime}\right)$ which satisfies the following two requirements: 1) If the nodes of $G$ have an $L$ type coloring using 3 colors, then the edges of $G^{\prime}$ have a $B$ type coloring using 3 colors; 2) If the edges of $G^{\prime}$ have a $B$ type coloring with 3 colors, then the nodes of $G$ have an $L$ type coloring with 3 colors.

Let $v_{1}, \cdots, v_{n}$ be all the vertices of $G$. This means that $V=\left\{v_{1}, \cdots, v_{n}\right\}$. We assign two points $w_{i}$ and $z_{i}$ to the node $v_{i}$ for each $i, 1 \leq i \leq n$. We choose the points $w_{1}, \cdots, w_{n}, z_{1}, \cdots, z_{n}$ to be pair-wise distinct. We connect the nodes $w_{i}$ and $z_{i}$. In other words $\left\{w_{i}, z_{i}\right\}$ is an edge of $G$ for each $i, 1 \leq i \leq n$. For each

$i, j, 1 \leq i<j \leq n$ we consider an isomorphic copy $L_{i, j}=\left(W_{i, j}, F_{i, j}\right)$ of the auxiliary graph $L=(W, F)$. If $v_{i}$ and $v_{j}$ are adjacent nodes in $G$, then we insert $L_{i, j}$ to $G^{\prime}$ such that the edge $\left\{w_{i}, z_{i}\right\}$ of $G^{\prime}$ is identified with the edge $\left\{x_{i, j, 1}, y_{i, j, 1}\right\}$ of $L_{i, j}$. Further the edge $\left\{w_{j}, z_{j}\right\}$ of $G^{\prime}$ is identified with the edge $\left\{\left(x_{i, j, 1}\right)^{\prime},\left(y_{i, j, 1}\right)^{\prime}\right\}$ of $L_{i, j}$. If $v_{i}$ and $v_{j}$ are not adjacent in $G$, then we do not add any new node or new edge to $G^{\prime}$.

We may say that the graph $G^{\prime}$ is a blown up version of the graph $G$. Each node of $G$ is replaced by two connected nodes in $G^{\prime}$. Further an isomorphic copy of the graph $L$ plays the role of each edge of $G$ in $G^{\prime}$. 
One can collapse the nodes $w_{i}$ and $z_{i}$ of $G^{\prime}$ to one point. If $\left\{w_{i}, z_{i}\right\}$ and $\left\{w_{j}, z_{j}\right\}$ are connected with an isomorphic copy $L_{i, j}$ of $L$, then one can collapse $L_{i, j}$ to a single edge. In this way one recovers the graph $G$ from the graph $G^{\prime}$.

Let us suppose first that the nodes of $G=(V, E)$ have an $L$ type coloring $f: V \rightarrow\{1,2,3\}$. We define an edge coloring $g: E^{\prime} \rightarrow\{1,2,3\}$ of $G^{\prime}=\left(V^{\prime}, E^{\prime}\right)$. We set $g\left(\left\{w_{i}, z_{i}\right\}\right)$ to be equal to $f\left(v_{i}\right)$. If $v_{i}$ and $v_{j}$ are adjacent nodes in $G$, then in the way we have seen in the proof of Proposition 4 we can extend the coloring of the edges $\left\{w_{i}, z_{i}\right\}=\left\{x_{i, j, 1}, y_{i, j, 1}\right\}$ and

$\left\{w_{i}, z_{i}\right\}=\left\{\left(x_{i, j, 1}\right)^{\prime},\left(y_{i, j, 1}\right)^{\prime}\right\}$ to each edge of the graph $L_{i, j}$. Thus the edges of $G^{\prime}$ have a $B$ type coloring as claimed in statement (1).

Let us suppose next that $g: E^{\prime} \rightarrow\{1,2,3\}$ is a $B$ type coloring of the edges of $G^{\prime}=\left(V^{\prime}, E^{\prime}\right)$. We define a coloring $f: V \rightarrow\{1,2,3\}$ of the nodes of $G$ by setting $f\left(v_{i}\right)$ to be equal to $g\left(\left\{w_{i}, z_{i}\right\}\right)$. We claim that $f\left(v_{i}\right)=f\left(v_{j}\right)$ and $i \neq j$ imply that $v_{i}$ and $v_{j}$ are not adjacent nodes in $G$. In order to prove the claim let us assume on the contrary that $f\left(v_{i}\right)=f\left(v_{j}\right), i \neq j$ and the nodes $v_{i}, v_{j}$ are adjacent in $G$. By Proposition $4, g\left(\left\{x_{i, j, 1}, y_{i, j, 1}\right\}\right) \neq g\left(\left\{\left(x_{i, j, 1}\right)^{\prime},\left(y_{i, j, 1}\right)^{\prime}\right\}\right)$ holds. On the other hand the definition of the coloring gives that $f\left(v_{i}\right)=g\left(\left\{w_{i}, z_{i}\right\}\right) \neq g\left(\left\{x_{i, j, 1}, y_{i, j, 1}\right\}\right)$. Similarly, by the definition of the coloring

$$
f\left(v_{i}\right)=g\left(\left\{w_{i}, z_{i}\right\}\right) \neq g\left(\left\{\left(x_{i, j, 1}\right)^{\prime},\left(y_{i, j, 1}\right)^{\prime}\right\}\right) \text {. From this }
$$

we get the contradiction $f\left(v_{i}\right) \neq f\left(v_{j}\right)$. This proves statement (2).

To complete the proof one should recall to the result that the problem of deciding if the nodes a given simple graph have a $L$ type coloring with 3 colors is an NPcomplete problem. Proofs of this well-known result can be found for example in $[6,7]$.

\section{The Derived Graph}

Let $G=(V, E)$ be a finite simple graph and suppose that we want to find a $B$ type coloring of the edges of $G$. A possible interpretation of the main result of this paper is that determining the optimal number of colors is a computationally demanding problem. In practical computations we should be content with suboptimal values of the number of colors.
From $G$ we construct a new graph $\Gamma=(U, F)$. The nodes of $\Gamma$ are the edges of $G$, that is, $U=E$. Two distinct nodes $\{x, y\}$ and $\{u, v\}$ of $\Gamma$ are connected in $\Gamma$ if $\{x, y\} \cap\{u, v\}=\varnothing$ and $x, y, u, v$ are not nodes of a 4-clique in $G$. In the lack of established terminalogy we call $\Gamma$ the derived graph of $G$. The essential connection between $G$ and $\Gamma$ is the following result.

Proposition 5. The nodes of $\Gamma$ have an $L$ type coloring with $k$ colors if and only if the edges of $G$ have a $B$ type coloring with $\mathrm{k}$ colors.

The reader will not have any difficulty to check the veracity of the proposition. The result makes possible to apply all the greedy coloring techniques developed for coloring the nodes of a graph. When the graph $G$ has n nodes it may have $O\left(n^{2}\right)$ edges. For instance when $G$ has 4000 nodes, then $G$ may have 10 millions edges. In this case the adjacency matrix of $\Gamma$ does not fit into the memory of an ordinary computer. Thus one should compute the entries of the adjacency matrix from the adjacency matrix of $G$ during the coloring algorithm. The most commonly used greedy coloring of the nodes of a graph takes $O\left(n^{2}\right)$ steps. Applying this technique to the derived graph we get an algorithm whose computational complexity is $O\left(n^{4}\right)$. The author has carried out a large scale numerical experiment with this algorithm. The results are encouraging.

\section{REFERENCES}

[1] I. M. Bomze, M. Budinich, P. M. Pardalos and M. Pelillo, "The Maximum Clique Problem," In: D.-Z. Du and P. M. Pardalos, Eds., Handbook of Combinatorial Optimization, Kluwer Academic Pubisher, Dordrecht, 1999, pp. 1-74.

[2] D. Kumlander, "Some Practical Algorithms to Solve the Maximal Clique Problem," Ph.D. Thesis, Tallin University of Technology, Tallinn, 2005.

[3] R. Carraghan and P. M. Pardalos, "An Exact Algorithm for the Maximum Clique Problem," Operation Research Letters, Vol. 9, No. 6, 1990, pp. 375-382. doi:10.1016/0167-6377(90)90057-C

[4] P. R. J. Östergård, "A Fast Algorithm for the Maximum Clique Problem," Discrete Applied Mathematics, Vol. 120, No. 1-3, 2002, pp. 197-207. doi:10.1016/S0166-218X(01)00290-6

[5] S. Szabó, "Parallel Algorithms for Finding Cliques in a Graph," Journal of Physics: Conference Series, Vol. 268, No. 1, 2011, Article ID: 012030. doi:10.1088/1742-6596/268/1/012030

[6] M. R. Garey and S. Johnson, "Computers and Intractability: A Guide to the Theory of NP-completeness," Freeman, New York, 2003.

[7] C. H. Papadimitriou, "Computational Complexity," Addison-Wesley Publishing Co. Inc., Boston, 1994. 